\title{
Alta frequência dos fatores de risco à oclusão dentária entre escolares no município de Petrópolis: um estudo transversal
}

\author{
High frequency of damaging factors to dental occlusion among \\ schoolchildren in the municipality of Petrópolis: a cross sectional study
}

Claudia Salvini Barbosa Martins da Fonseca1, Maria de Fátima Pombo March², Lilian Teresinha Costa1,3,4,

Clemax Couto Sant'Anna ${ }^{4}$

Faculdade Medicina de Petrópolis (FMP) - Petrópolis (RJ), Brasil.

Instituto de Puericultura e Pediatria Martagão Gesteira (IPPMG), Universidade Federal do Rio de Janeiro (UFRJ) - Rio de Janeiro (RJ), Brasil.

${ }^{3}$ Programa de Pós-Graduação em Biotecnologia, Inmetro - Duque de Caxias (RJ), Brasil.

${ }^{4}$ Universidade Federal do Rio de Janeiro (UFRJ) - Rio de Janeiro (RJ), Brasil.

DOI: https://dx.doi.org/10.7322/abcshs.v44i1.1086

\begin{abstract}
RESUMO
Introdução: A má oclusão dentária tem origem multifatorial, logo é difícil definir estratégicas específicas de como preveni-la. A respiração bucal e certos hábitos de sucção, se persistir por mais de 36 meses, podem influenciar de forma negativa. Objetivo: Identificar fatores de risco à oclusão dentária como: respiração bucal e hábitos de sucção em escolares de 8 a 10 anos de idade em Petrópolis, RJ. Métodos: Estudo observacional, transversal, de caráter descritivo. Incluídos escolares de ambos os sexos, 8 a 10 anos, matriculados em sete escolas municipais de Petrópolis. Distribuído aos escolares o protocolo para a identificação da criança respiradora bucal e o questionário sobre hábitos de sucção (mamadeira, chupeta e dedo). Resultados: Foram avaliados 377 protocolos para a identificação de criança respiradora bucal e 377 questionários sobre hábitos de sucção. Nos protocolos foram referidos sintomas como: dormir de boca aberta em 193 $(51,2 \%)$, babar no travesseiro em $172(45,6 \%)$, roncos em 131 $(34,7 \%)$ e obstrução nasal diária em $118(31,2 \%)$. Ao aplicar os critérios de Abreu, constatou-se uma frequência de 243 (64\%) respiradores bucais e 134 (36\%) respiradores nasais. Ao avaliar os 377 questionários sobre os hábitos de sucção encontraram-se: 276 (73\%) crianças com hábitos de sucção e 101 (27\%) sem tais hábitos. Dos 276 escolares que tiveram hábitos de sucção, houve persistência destes hábitos acima de três anos e 11 meses em 149 crianças (54\%). Conclusão: Encontrada uma alta frequência de respiradores bucais e de crianças com hábitos de sucção.
\end{abstract}

Palavras chaves: criança; má oclusão; respiração bucal; comportamento de sucção.

\begin{abstract}
Introduction: Dental malocclusion has a multifactorial origin, so it is difficult to define specific strategies for preventing it. Mouth breathing and certain sucking habits, if they persist for more than 36 months, can have a negative influence. Objective: To identify potentially damaging factors to dental occlusion like mouth breathing and sucking habits in children aged 8 to 10 years old in Petrópolis, RJ. Methods: Cross-sectional, observational, descriptive study. Both male and female children between the ages of 8 to 10 years, enrolled in seven public schools of Petrópolis, were included. The identification protocol for mouth breathing child and the questionnaire on sucking habits (feeding bottle, pacifier and finger sucking) were applied to the students. Results: A total of 377 protocols for identification of mouth breathing children and 377 questionnaires for suction habits were evaluated. The following symptoms were reported: open mouth sleeping in 193 (51.2\%), drooling on the pillow in 172 (45.6\%), snoring in 131 (34.7\%) and daily nasal obstruction in $118(31,2$ $\%)$. After application of Abreu criteria, a frequency of $243(64 \%)$ mouth breathers and 134 (36\%) nasal breathers was observed. Evaluation of the questionnaires about sucking habits demontrated 276 (73\%) children with sucking habits and 101 (27\%) without such habits. Of the 276 schoolchildren with sucking habits, those habits persisted for more than 3 years and 11 months in 149 children (54\%). Conclusion: A high frequency of mouth breathing and sucking habits children were found.
\end{abstract}

Keywords: child; malocclusion; mouth breathing; sucking behavior.

Recebido em: 29/11/2017 


\section{INTRODUÇÃO}

O pediatra, pelo seu frequente convívio com a criança, está em uma posição única em relação à observação e prevenção de uma má oclusão dentária, para tal é necessário estar ciente de fatores que possam contribuir neste processo ${ }^{1,2}$.

Nos primeiros anos de vida, a face do lactente é relativamente larga e curta, o nariz é pequeno, os olhos relativamente distantes e a testa bem proeminente. Nesta fase, o complexo nasomaxilar e a mandíbula são menores que o neurocrânio. Com o crescimento, devido a uma maior demanda do sistema respiratório e mastigatório, ocorre um grande crescimento do complexo facial que é composto pelo nariz, mandíbula e maxila ${ }^{3}$.

O sistema estomatognático é muito citado pelos fonoaudiólogos e odontólogos, mas pouco conhecido dos pediatras. Este sistema é composto pelos ossos da mandíbula e maxilar assim como o zigomático, temporal, esfenoide, hioide, coluna cervical e a toda a neuromusculatura que mobiliza estes ossos entre si. A respiração, sucção, deglutição, mastigação e fonoarticulação são atividades exercidas por este sistema. A atividade muscular exercida em cada uma destas funções desempenha um vetor de crescimento direcionando o crescimento ósseo craniofacial ${ }^{4}$.

Os fatores que favorecem um crescimento harmônico deste sistema são aqueles que por meio da estimulação adequada da musculatura impulsionam o crescimento craniofacial corretamente como: aleitamento materno, deglutição adequada, mastigação de alimentos sólidos e a respiração nasal ${ }^{5}$.

Fatores que podem contribuir para um crescimento desarmônico são: características hereditárias, anomalias congênitas, traumas, perda prematura de um dente, alimentos pastosos, interposição de língua ou do lábio inferior entre a arcada superior e a inferior assim como doenças sistêmicas ou locais, respiração bucal e os hábitos de sucção se persistirem após a idade de quatro anos de idade ${ }^{1,5,6}$.

Um crescimento craniofacial harmônico predispõe a uma boa oclusão dentária. Podemos definir uma oclusão ideal como aquela na qual a relação dos dentes da arcada superior e inferior apresente o menor stress à articulação temporomandibular, uma ótima função do complexo orofacial, boa estabilidade e estética facial ${ }^{1}$.

Já a má oclusão dentária tem origem multifatorial, logo é difícil definir estratégicas específicas de como preveni-la. Mas estamos cientes que a respiração bucal e certos hábitos orais como o uso de chupeta, mamadeira ou dedo se persistirem por mais de 36 meses podem influenciar de forma negativa o crescimento maxilo-mandibular, comprometendo o crescimento das arcadas superior e inferior e, consequentemente predispondo a uma má oclusão ${ }^{1,2,6}$.

Nas patologias referidas acima, ocorre um desbalanço das forças entre a musculatura interna (língua) e a musculatura externa (lábios e bochechas). Nos hábitos de sucção há uma alteração importante no padrão de deglutição: a criança ao deglutir empurra a língua para frente. Já na respiração oral, ocorre uma hipotonia do musculo bucinator (não há vedamento labial), a língua repousa no assoalho da boca e há hipertonicidade do musculo bucinador ${ }^{1,7}$.

O pediatra ao observar a arcada dentária pode ser capaz de identificar uma oclusão ideal assim como suspeitar de uma má oclusão $^{1}$ (mordida aberta, mordida profunda, overjet, mordida cruzada, topo a topo e apinhamento) (Figura 1).

A recomendação atual da American Academy of Pediatric Dentristy (AAPD) é que seja realizado um acompanhamento odontológico desde a gestação, sendo orientado à mãe uma dieta adequada, hábitos de limpeza oral, tratamento dentário e instruções sobre a saúde bucal da criança ${ }^{2}$. O odontopediatra deve acompanhar desde o inicio da erupção da primeira dentição da criança, sempre atento à prevenção de cárie e à presença de fatores que possam desencadear deformações dentoalveolar ou esqueléticas.

Desta forma, quanto mais cedo for detectada uma alteração mais rapidamente poderá ser feita uma intervenção. O objetivo é minimizar a ortodontia corretiva, favorecendo a interceptação da má oclusão antes do seu estabelecimento. Trata-se de tratamentos interceptativos, os quais podem ser realizados pelo odontopediatra. Esses procedimentos são menos invasivos ${ }^{6}$.

Mesmo em países desenvolvidos poucas crianças seguem as recomendações acima, assim sendo o pediatra ao suspeitar de uma alteração deverá encaminhar ao odontopediatra, pois a prevenção de uma má oclusão tem um grande impacto na vida deste indivíduo ${ }^{2,8}$.

O objetivo desse estudo foi identificar a presença de potenciais fatores prejudiciais à oclusão dentária como: respiração bucal e alguns hábitos de sucção (mamadeira, chupeta e dedo) em escolares de oito a 10 anos de idade no município de Petrópolis.

\section{MÉTODOS}

Estudo observacional, transversal, descritivo. Foram selecionados escolares de ambos os sexos, nas faixas etárias de 8 a 10 anos



Figura 1: Tipos de má oclusão dentária 
de idade, matriculados em sete escolas municipais de Petrópolis, RJ. Realizada a entrega e coleta do questionário e protocolo no período de fevereiro a maio de 2016. Foi escolhida esta faixa etária por apresentarem uma dentição mista, período propício a intervenções para o tratamento de oclusões dentárias. Petrópolis é uma cidade serrana com clima tropical de altitude, com verões úmidos e chuvosos e invernos secos e frios. O critério de inclusão foi: escolares matriculados em sete escolas municipais próximas ao centro da cidade com pouca variação climática entre elas. Foram excluídas as crianças com doenças genéticas com comprometimento facial, doenças com envolvimento musculoesqueléticas e formulários incompletos.

Foi distribuído aos escolares o protocolo para a identificação da criança respiradora bucal de Abreu et al. ${ }^{9}$ Concomitantemente foi distribuído um questionário sobre a presença ou não de hábitos de sucção, como mamadeira, chupeta e dedos e se estes hábitos persistiram até três anos e 11 meses ou se ultrapassaram esta idade. $\mathrm{O}$ protocolo e o questionário são compostos com as variáveis deste estudo e foram respondidos pelos responsáveis. Foram consideradas respiradoras bucais (RBs), as crianças que apresentavam dois sinais maiores (roncar, dormir com a boca aberta, babar no travesseiro, queixar-se de obstrução nasal diária) ou um sinal maior associado a dois ou mais sinais menores (prurido nasal, obstrução intermitente, às vezes referir dificuldade para respirar à noite ou sono agitado, sonolência, irritabilidade diurna, dificuldade ou demora ao engolir alimentos, três ou mais episódios de infecção de garganta ou ouvido, ou sinusite comprovada por médico no último ano, dificuldade no aprendizado escolar. As demais foram consideradas respiradoras nasais (RNs).

O projeto foi aprovado pelo Comitê de Ética Médica da Faculdade Medicina de Petrópolis, sob o número CAAE 51737915.1.0000.5245.

\section{RESULTADOS}

Foram distribuídos 585 protocolos de anamnese e questionários sobre hábitos de sucção, destes foram devolvidos 447, mas 70 foram excluídos (em 38 a idade não era compatível com a faixa etária do trabalho, 19 estavam com as respostas incompletas e 13 sem a assinatura dos responsáveis). Ao final, foram analisados 377 protocolos e questionários (Figura 2).

A amostra era composta por 174 escolares do sexo masculino e 203 do sexo feminino, distribuídos segundo a faixa etária: oito anos de idade (120 escolares), nove anos (151) e 10 anos de idade (106).

Ao analisar os protocolos foi observada uma alta frequência de sintomas como: dormir de boca aberta em 193 (51,2\%), babar no travesseiro em 172 (45,6\%), roncos em 131 (34,7\%) e obstrução nasal diária em 118 escolares (31,2\%). Sugestivos de uma possível obstrução e ou mudança no padrão de respiração.
Ao aplicar os critérios de Abreu et al. ${ }^{9}$, dividiu-se esta amostra em 243 (64\%) RBs e 134 (36\%) RNs e, foi observado que certos sintomas foram bem mais frequentes nos RBs do que nos RNs tais como: dormir de boca aberta em $73,6 \%$ versus $10,4 \%$, salivação no travesseiro em $70,7 \%$ versus $12,6 \%$, roncos em $53,9 \%$ versus $6,71 \%$, obstrução nasal diária em $48,5 \%$ versus $4,4 \%$, sonolência diurna em $19,7 \%$ versus $2,2 \%$, dificuldade de respirar/sono agitado em $47,7 \%$ versus $8,2 \%$, dificuldade de engolir alimentos em $19,3 \%$ versus $3,7 \%$ (Tabela 1 ).

Ao avaliar os questionários sobre os hábitos de sucção (mamadeira, chupeta e dedo) constatou-se que estes hábitos foram bem frequentes: 276 (73\%) crianças com algum hábito de sucção (Figura 3) e 101 (27\%) sem tais hábitos.

Dos 276 escolares que apresentaram algum hábito de sucção, houve persistência destes hábitos acima de três anos e 11 meses em 149 crianças (54\%). Destas 149 crianças: 98 escolares $(65,8 \%)$ eram RBs e 51 escolares $(34,2 \%)$ eram RNs.

\section{DISCUSSÃO}

Foi encontrada uma elevada prevalência de RBs (65\%) nesta amostra. Outros estudos realizados em outras cidades brasileiras encontraram uma prevalência de 55\% em Abaeté e 56,8\% em Londrina ${ }^{9,10}$.

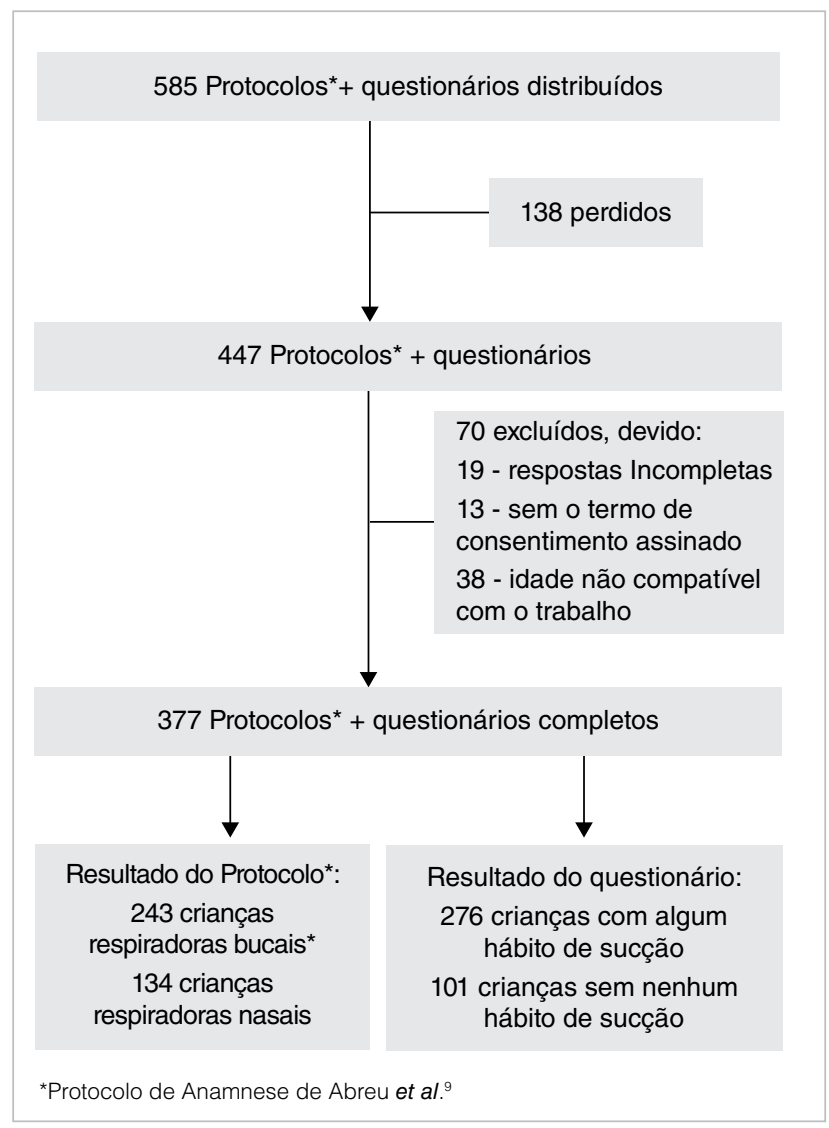

Figura 2: Desenvolvimento do estudo a partir do protocolo e questionário distribuídos. 
No dia a dia do pediatra uma grande parcela dos responsáveis pelas crianças, durante a consulta pediátrica, não refere se a criança apresenta algum distúrbio do sono como: dormir de boca aberta, roncos, apneia ou mesmo não valoriza a qualidade de sono. Baseado nestes dados, a Academia Americana de Pediatria (AAP) a fim de fazer uma triagem para a apneia do sono recomenda que se pergunte a todo responsável da criança ou adolescente se este ronca ${ }^{11}$. Observamos que na população de RBs, os distúrbios do sono como: dormir de boca aberta estava presente em quase três quartos e os roncos na metade dos entrevistados.

Comparando um grupo com o outro, observou-se que os roncos foram oito vezes mais frequentes nos RBs que nos RNs assim dormir de boca aberta sete vezes. Confirmando como é valioso o pediatra investigar sobre o sono durante a anamnese e, a partir destes dados procurar ativamente durante o exame físico sinais compatíveis com obstrução.

Há estudos que correlacionam a respiração bucal a possíveis alterações na arcada dentária e, consequentemente a algum tipo de má oclusão $0^{6,7,12,13}$. Porém é importante enfatizar que a hereditariedade apresenta um papel importante no crescimento e desenvolvimento facial ${ }^{14}$.

Apesar de não ser o foco da discussão, vale ressaltar que sintomas como: sonolência diurna, dificuldade de respirar à noite/sono agitado e dificuldade escolar foram mais frequentes nos RBs. Estes resultados são concordantes com os estudos realizados na coorte de Avon, nos quais houve relação entre distúrbios do sono e alteração da cognição e desenvolvimento da linguagem, bem como distúrbios do sono e alteração do comportamento ${ }^{15,16}$.

Em relação ao questionário sobre hábitos de sucção, na nossa amostra quase $3 / 4$ das crianças apresentavam pelo menos algum hábito. Dependendo de sua frequência, intensidade e duração podem estar associadas com deformações dentoalveolares ou esquelética ${ }^{6}$.
No nosso estudo, mais da metade dos nossos entrevistados tiveram, em algum momento, o hábito de utilizar a mamadeira. Há estudos que não encontraram associação entre o uso de mamadeira e má oclusão ${ }^{17,18}$, enquanto Moimaz et al. ${ }^{19}$ citam esta associação na primeira dentição. Futuros estudos longitudinais serão necessários para melhor esclarecimento. A AAP recomenda que a criança que utiliza mamadeira faça a transição para o copo na idade de 12 a 24 meses $^{20}$.

Quase a metade dos escolares desse estudo, em algum momento, teve o hábito de usar a chupeta. Sobre a associação entre chupeta e má oclusão, um estudo sueco com 457 crianças de três anos de idade correlacionou o uso de chupeta e o dedo com má oclusão na primeira dentição ${ }^{21}$. É frequente os pais oferecerem a chupeta à criança para acalma-la ${ }^{22}$, apesar de estarem cientes que a presença e manutenção de hábitos orais deletérios alteram as funções

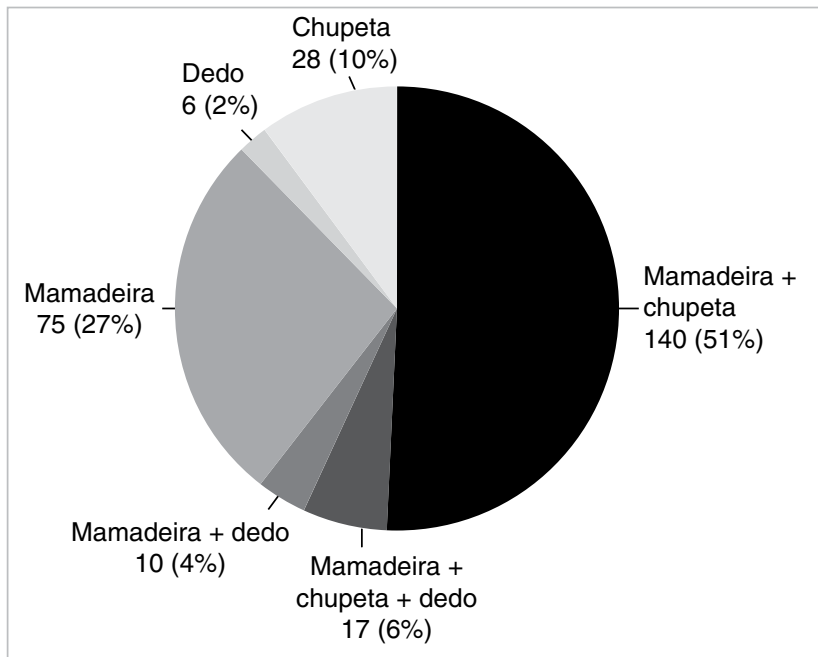

Figura 3: Distribuição dos hábitos de sucção.

Tabela 1: Sintomas referidos pelos respiradores bucais e nasais.

\begin{tabular}{|l|c|c|c|c|c|c|}
\hline Sintomas & $\begin{array}{c}\text { Respirador } \\
\text { bucal }\end{array}$ & $\begin{array}{c}\text { Respirador } \\
\text { nasal }\end{array}$ & $\begin{array}{c}\text { Risco } \\
\text { prevalência }\end{array}$ & IC 95\% & $\begin{array}{c}\text { Odds } \\
\text { ratio }\end{array}$ & IC 95\% \\
\hline Total & 243 & 134 & & & & \\
\hline Ronca & 131 & 9 & 8,02 & $6,33-9,71$ & 16,25 & $7,89-33,43$ \\
\hline Dorme boca aberta & 179 & 14 & 7,05 & $5,56-8,53$ & 23,97 & $12,86-44,70$ \\
\hline Baba no travesseiro & 172 & 17 & 5,57 & $4,40-6,75$ & 16,67 & $9,34-29,75$ \\
\hline Obstrução nasal diária & 118 & 6 & 10,84 & $8,55-13,12$ & 20,14 & $8,55-47,43$ \\
\hline Prurido nasal & 99 & 14 & 3,89 & $3,07-4,72$ & 5,89 & $3,20-10.85$ \\
\hline Obstrução intermitente & 204 & 68 & 1,65 & $1,30-2,00$ & 5,07 & $3,13-8,22$ \\
\hline Dificuldade de respirar a noite/ sono & 116 & 11 & 5,81 & $4,58-7,04$ & 10,21 & $5,24-19.89$ \\
\hline agitado & 48 & 3 & 8,82 & $6,96-10,68$ & 10,74 & $3,27-35,24$ \\
\hline Sonolência diurna & 101 & 20 & 2,78 & $2,19-3,37$ & 4,05 & $2,36-6.95$ \\
\hline Irritabilidade diurna & 47 & 5 & 5,18 & $4,09-6,27$ & 6,18 & $2,39-15,97$ \\
\hline Dificuldade engolir alimentos & 81 & 14 & 3,19 & $2,51-3,86$ & 4,28 & $2,318-7,924$ \\
\hline Mais de 3 episódios infecção/ano & 82 & 11 & 4,111 & $3,24-4,97$ & 5,69 & $2,909-11,15$ \\
\hline Dificuldade escolar & & & & &
\end{tabular}


do sistema estomatognático como a oclusão, respiração e fala ${ }^{23}$. A maior persistência nos hábitos de sução não nutritivos está associada à má oclusão ${ }^{24}$. O uso de chupeta ortodôntica é preferível ao hábito de sucção do dedo, pois este é mais difícil de ser controlado pelos pais ${ }^{18}$. Portanto, a chupeta deve ser desencorajada e, a idade limite seria de quatro anos ${ }^{24}$. Na nossa amostra o hábito de utilizar a chupeta persistiu em quase metade das crianças após quatro anos.

Este estudo tem suas limitações por não utilizar questionários validados e pelo fato dos questionários terem sido respondidos pelos responsáveis. Teria sido oportuno ter realizado o exame físico destas crianças pesquisando a presença de má oclusão dentária.

O pediatra ao identificar uma alteração no padrão respiratório deve identificar a etiologia e tratá-la e, ao constatar a presença de hábitos de sucção deve orientar a família quanto à necessidade da retirada destes de uma forma tranquila para a criança. Encaminhar o paciente a uma equipe multidisciplinar com um alergista, otorrino, odontopediatra e fonoaudiólogo, irá assegurar a eliminação destes fatores nocivos permitindo um crescimento harmônico.

Conclui-se que, foi encontrada uma alta prevalência de respiradores bucais e de crianças com hábitos de sucção.

\section{AGRADECIMENTOS}

Os autores agradecem a valorosa ajuda de Fernando Stockler e Adriana Tavares Gomes Gonçalves.

\section{REFERÊNCIAS}

1. Emerich K, Wojtaszek- Stominska A. Later orthodontic complications caused by risk factors observed in the early years of life. Eur J Pediatr 2010;169(6):651-5. http://dx.doi.org/10.1007/s00431-009-1098-6

2. American Academy of Pediatric Dentristy (AAPD). Guideline on perinatal and infant oral health care. Pediatr Dent. 2016;38(6):150-4

3. Kozak FK, Ospina JC, Cardenas MF. Characteristics of normal and abnormal postnatal craniofacial growth and development. In: Lesperance MM, Flint PW. Cummings pediatric otolaryngology. Elsevier, 2015; p. 55-80.

4. Souza LBR. A atuação da fonoaudiologia na oclusão neutral, nas más oclusões e nas DTMs. In: Paiva HJ. Noções e conceitos básicos em oclusão, disfunção temporomandibular e dor orofacial. São Paulo: Santos; 2008. p.67-78.

5. Capellete Junior M. Tratamento das consequências. In: Solé D, Prado E, Wecky LLM. Obstrução nasal: o direito de respirar pelo nariz. São Paulo: Atheneu, 2013; p. 233-47.

6. American Academy of Pediatric Dentristy. Guideline on management of the developing dentition and occlusion in pediatric dentristy. Pediatr Dent. 2016;38(6):289-301.

7. Grippaudo C, Paolantonio EG, Antonini G, Saulle R, La Torre G, Deli R. Association between oral habits, mouth breathing and malocclusion. Acta Otorhinolaryngol Ital. 2016;36(5):386-94. http://dx.doi.org/10.14639/0392-100X-770

8. Peres KG, Chaffee BW, Feldens CA, Flores-Mir C, Moynihan $P$, Rugg-Gunn A. Breastfeeding and oral health: evidence and methodological challenges. J Dent Res. 2018;97(3):251-8. http://dx.doi.org/10.1177/0022034517738925

9. Abreu RR, Rocha RL, Lamounier JA, Guerra AFM. Etiology, clinical manifestations and concurrent findings in mouth breathing children. J Pediatr. 2008;84(6):529-35. http://dx.doi.org/10.1590/S0021-75572008000700010

10. Felcar JM, Bueno IR, Massan ACS, Torezan RP, Cardoso JR. Prevalência de respiradores bucais em crianças de idade escolar. Ciênc Saúde Coletiva. 2010;15(2):427-35. http://dx.doi.org/10.1590/S1413-81232010000200020
11. Marcus CL, Brooks LJ, Draper KA, Gozal D, Halbower AC, Jones $J$, et al. Diagnosis and management of childhood obstructive sleep apnea syndrome. Pediatrics. 2012;130(3):e714-55. http://dx.doi.org/10.1542/peds.2012-1672

12. Lione $\mathrm{R}$, Buongiorno M, Franchi L, Cozza P. Evaluation of maxillary arch dimensions and palatal morphology in mouth breathing children by using digital dental casts. Int J Pediatr Otorhinolaryngol. 2014;78(1):91-5.

http://dx.doi.org/10.1016/j.ijporl.2013.09.028

13. Imbaud TCS, Mallozi MC, Domingos VBTC, Solé D. Frequência de rinite e alterações orofaciais em pacientes com má oclusão dentária. Rev Paul Pediatr. 2016;34(2):184-8. http://dx.doi.org/10.1016/j.rppede.2016.02.009

14. Souki BQ, Pimenta GB, Souki MQ, Franco LP, Becker HMG, Pinto $J A$. Prevalence of malocclusion among mouth breathing children: do expectations meet reality? Int J Pediatr Otorhinolaringol. 2009;73(5):767-73 http://dx.doi.org/10.1016/j.ijporl.2009.02.006

15. Bonuck K, Rao T, Xu L. Pediatric sleep disorders and special educational need at 8 years: a population based cohort study Pediactrics. 2012;130(4):634-42.

http://dx.doi.org/10.1542/peds.2012-0392

16. Bonuck K, Freeman K, Chervin RD, Xu L. Sleep disorders breathing in a population based cohort: behavioral outcomes at 4 and 7 years. Pediactrics. 2012;129(4):e857-65. http://dx.doi.org/10.1542/peds.2011-1402

17. Hermont AP, Martins CC, Zina LG, Auad SM, Paiva SM, Pordeus IA. Breastfeeding, bottle feedings and malocclusion in the primary dentition: a systematic review of cohort studies. Int J Environ Res Public Health. 2015;12(3):3133-51. http://dx.doi.org/10.3390/ijerph120303133

18. Abreu LG, Paiva SM, Pordeus IA, Martins CC. Breastfeeding, bottle feedings and malocclusion in mixed and permanent dentitions: a systematic review. Braz Oral Res. 2016;30:e22. http://dx.doi.org/10.1590/1807-3107BOR-2016.vl30.0022

19. Moimaz SA, Garbin AJ, Lima AM, Lolli LF, Saliba O, Garbin CA Longitudinal study of habits leading to malocclusion development in childhood. BMC Oral Healthy. 2014:14:96. http://dx.doi.org/10.1186/1472-6831-14-96 
20. American Academy of Pediactrics. Pacifiers and thumb sucking. Disponível em: http://www.healthychildren.org/english/agesstages/baby/feeding-nutrition/pages/discontinuing-the-bottle. Acesso em: 27 maio 2017.

21. Dimberg L, Bondemark L, Soderfeldt B, Lennartsson B. Prevalence of malocclusion traits and sucking habits among 3 years old children. Swed Dent J. 2010;34(1):35-42.

22. Garbin CAS, Garbin AJI, Martins RJ, Souza NP, Moimaz SAS. Prevalência de hábitos de sucção não nutritivos em pré escolares e a percepção dos pais sobre a sua relação com maloclusões. Ciênc Saúde Coletiva. 2014;19(2):553-8.

http://dx.doi.org/10.1590/1413-81232014192.23212012

23. Majorana A, Bardellini E, Amadori F, Conti G, Polimeni A. Timetable for oral prevention in childhood developing dentition and oral habits: a current opinion. Prog Orthod. 2015;16:39.

http://dx.doi.org/10.1186/s40510-015-0107-8

24. Sexton S, Natale R. Risks and benefits of pacifiers. Am Fam Physician. 2009;79(8):681-5. 